$\Rightarrow$ SMALL RNAS

\title{
A novel class
}

Recent profiling studies have identified many new types of small RNA, indicating a greater degree of transcriptome complexity than previously suspected. Now, an unusual class of small RNAs has been discovered - antisense termini-associated short RNAs (aTASRs) - that might be copied from mRNAs.

Kapranov and colleagues used true single-molecule sequencing of first-strand cDNA to profile transcripts in human cells and found a novel class of small RNAs that map to the termini of annotated genes and are on the antisense strand. These aTASRs were present at many genes, and the genes flanked by these novel RNAs were enriched for translation-related functions, which could provide clues about the potential biological roles of aTASRs.

The aTASRs have a non-genomically encoded 5' poly(U) tail and start almost exactly at the end of the transcripts that they are antisense to. The authors suggest that these features are consistent with a mechanism in which an RNA-dependent RNA polymerase (RdRP) could generate these transcripts by copying polyadenylated mRNAs. Recently, human telomerase reverse transcriptase was shown to have RdRP activity, and an enzyme with RdRP activity has been identified in flies, so it is possible that other RdRPs could also exist. Future studies will be required to determine how common these novel RNAs are, what their functions are and the mechanism by which they are generated, but this study has provided an intriguing starting point. 